\title{
Modelling Techniques for Benchmarking in Complex Administration Systems: an Approach from a Total Quality Viewpoint
}

\author{
Walter Ukovich \\ Franca Zerilli \\ DEEI \\ IT Consultant \\ University of Trieste \\ phone: +39406767135 \\ fax: +39406763460 \\ e.mail: ukovich@univ.trieste.it
}

\begin{abstract}
This paper deals with enterprise modelling and benchmarking techniques used in a typical non-profit environment, such as a State University in Italy.

Moving from the need to evaluate efficiency and effectiveness of the University as a whole, a frame of reference has firstly settled down to describe the as is state of the service supply, to organize and manage the evaluation of efficiency and effectiveness in the central administration and to examine the problems related to the performance inprovement in order to fulfill quality policies or to meet external standards.

The aim of the contribution is to point out the effectiveness and the opportunities provided by some Information Engineering formal representation techniques in setting up and then maintaining models that, first of all, represent business processes needed to provide services and then help in approaching and managing the benchmarking activities.

This work is part of a larger project that, from a total quality point of view, has as a final scope to define strategies and guidelines for quality service supply both in the administrative, teaching and research contexts.

The technique and the models presented can be used both for the quality features definition, in order to fulfill end-user quality expectations, and to set up the maintenance activities - from a supplier and an end-user point of view - also by using benchmarking techniques.
\end{abstract}

Keywords benchmarking - modelling techniques - public administration - quality of services - university services 


\section{Introduction}

In November of 1993 the University of Trieste, Italy has started a six-month project devoted to define guidelines and strategic objectives for the production of quality services by the University Central Administration.

The University of Trieste is a medium-size (according to the Italian figures) state University with about 1,250 professors for over 22,000 students in the present Academic Year, 70 Departments and ten Faculties.

This project has been denominated SQUADRA (a multi-meaning Italian word corresponding to "setsquare", "squad" and "team") as an acronym of the motto "Quality Services: a Decision Support for the Head of the University". To date, the first phase of the project has been accomplished, which was devoted to specifying the general approach and methodology for the whole project, and to analize the system of interest. A formal model of the Central Administration of the University of Trieste has been produced and validated.

The scope of this paper is to present the aspects of the SQUADRA project, and in particular the results which bear some relevance for possible benchmarking activities in the specific area of the University Administration, and, more in general, in the wider framework of complex systems providing services.

\section{Benchmarking}

The benchmarking technique is used to evaluate business processes in an organization through the continuous comparison of its performance parameters with the parameters of other leading competitors, both in similar and in different business areas. The aim in using benchmarking is:

- to spot out weak points and critical issues;

- to scout out opportunities of improvement;

- to plan actions directed to achieve improvements through a process management approach

The benchmarking activities may be structured in phases. Taking a slightly more general approach then usual, they can be described as follows:

1. analysis of the system: a formal model of the system (or of part of it) under evaluation is formulated;

2. design of the measurements: the relevant parameters for the evaluation are identified and the measurement sessions are programmed; also, other organizations to be used for comparisons are selected; furthermore, whenever possible, appropriate activities directed to acquire other qualitative informations about the the other organizations, such as structure, functions, procedures, and even history; 
3. measurement activities: the parameters of the previous point are measured; furthermore, whenever possible, the other qualitative informations about the other organizations, as mentioned in the previous step, are also studied;

4. comparisons: the measured parameters are compared in order to detect weaknesses, drawbacks, bottlenecks, and to assess target levels for improvement; furthermore, the qualitative information gathered in the previous phase are used to figure out possible ways of improving the performace of the system;

5. formulation of proposals: on the base of the comparisons and of the other information acquired in the previous phase, operational action proposals are formulated, aimed to raise the performance levels of the system to the target values.

\subsection{Remarks}

The role of the phase of analysis must not be underestimated, although it is often not explicitly mentioned; in fact, a suitable model for the system of interest is often available, and the nature and structure of the relevant processes are clear and well known. However, this is not always the case and italian governament supported organizations, expecially those providing services, are quite good examples of situations in which more attention is devoted to the procedural aspects of production rather than on how each production step is combined with the others in order to form the business processes.

In such situations building and using an effective model could constitute a key success factor for using benchmarking techniques.

According to our opinion, the model of the system resulting from the analysis phase must be implementation free, that is it must describe the process of interest in conceptual terms, focusing on what is done rather than on how it is done. In this way a wider spectrum of opportunities is guaranteed to find alternative possibilities of implementing the process in order to achieve the desired performance levels.

In the design phase, the operational characteristics of the process are added to the model, which becomes in this way a more faithful representation not only of the specific process of interest, but also of the implementation choices characterizing the considered situation. In this phase the model evolves from a preminently qualitative formulation to a preminently quantitative one, thus providing the basis for identifying the benchmarking parameters.

Concerning the phase of measurement, it must be pointed out that, according to our views, it consists of two different kinds of activities: quantitative evaluation of the relevant parameters, and retrieval of further qualitative information about the comparison organizations.

The latter activity can be interpreted as a modelling activity on the other organizations, which is conceptually analogous to the modelling activity of the analysis phase. Having used an implementation-free model there can be useful also in this aspect of the measurement phase, since, at least in principle, the same approach could be used. Of course, this could be utopistic in most competitive environments, 
such as in industrial business, but could be very effective in cooperative situations, where several organizations cooperate in an integrated benchmarking project.

\section{Contribution of the SQUADRA project to bench- marking modelling techniques}

In this section we analyze the contribution of the SQUADRA project within the framework of the benchmarking, as it has been discussed in the previous section.

The aim of the SQUADRA project is to study how to fulfill the perceived (and the imposed) needs of evaluating the efficiency and effectiveness of the processes in the University of Trieste, Italy, and in particular of the central administration of it.

To this end, a quality based approach has been taken in order to identify the services provided and the processes generating them. It is important to point out that defining guidelines and strategic objectives concerning quality constitutes an important part in the organization management policies [5], [6]. According to this approach, the service users and the transversal (with respect to the vertical functional lines) flow of activities producing services within the system have been particularly focused on.

The results produced so far in the SQUADRA project pertain to the phase of analysis of the benchmarking technique. They are an example of the application of modelling techniques drawn from Information Technology environments [7], to a complex administrative system. These models are being used with a view to identifying measurable parameters representing the quality features of the system (or part of it), considered as a complex of business processes.

\section{Scenario of the SQUADRA project}

Universities in Italy, and also in the rest of Europe, must face in the near future situations of increasing complexity with decreasing available resources but tighter competition levels and higher expectations.

This is true for private educational institutions, but also for government supported universities, due to the specific situation of the Italian state economy.

As a consequence, Universities in Italy begin to realize that it is time to define, design and implement tools apt to evaluate efficiency and effectiveness about the use of resources they have. Several committments in this sense have been formulated, both at the national and European levels.

Universities are considered as organizations providing cultural services. The activities within them belong to one of the three areas of teaching, research, and administration. The two former ones are primary activities, in the sense that they directly contribute to the fulfillment of the institutional objectives, while the latter one just supports them.

The part fo the SQUADRA project here presented is devoted to study the administration of the University of Trieste in order to approach efficiency and effectiveness evaluation problems and formulate action proposals to organize and manage 
them and the performance improvement needed to fulfill internal quality policies or to meet external standards.

\section{Motivations and general approach of the project}

Roughly speaking, the concepts of efficiency and effectiveness evaluation immediately refer to the idea of measuring features of the relevant product or service, and to elements such as performance evaluators, types of measurements, resource assignment criteria, and so on.

According to our opinion, such aspects, although important, must be embedded within a general framework in order to guarantee that some relevant requirements are met:

- evaluations in different application environments must be performed according to a uniform approach; this requires that they are all conceived and designed within a unique conceptual framework;

- evaluation techniques must be context-free and therefore valid in different application environments;

- it must be possible to iterate evaluations, if necessary, in the same application environment in order to follow its time evolution; moreover, sinergies must result from performing similar evaluations in different application environments; in this respect, result comparability is a necessary feature:

- evaluating must facilitate diagnosing detected inefficiencies and ineffectivenesses in terms of internal coherece of the measured parameters.

In order to show how our approach to evaluating services of the University is able to meet such requirements, we first need to formally specify some concepts.

Definition $1 A$ service is the effect of the concurrent execution of some functional steps within the organization, connecting an "activator", who triggers the whole precess, to a "client", who gets the products yielded by the process.

Note that we only consider here the production phase of the whole service lifecycle.

According to the previous definition, we distinguish external and internal services, depending upon the relative position of activators and clients with respect to the structure providing the service. For instance, services to students are external, while services related to the management of academic status of professors are internal.

The key choice of the SQUADRA project is the quality-based approach to services [1], [2]. This allows to focus on the interaction between the system producing services and its environment, in particular its users, and their expectations on the products provided by the system.

From this quality-based approach we can identify what has to be considered acceptable as a product of the system. As a consequence, we can also fix the 
guidelines according to which the features of the system that are relevant with respect to efficiency and effectiveness issues can be assessed.

The normative framework of the SQUADRA project is given by the European Standards EN 29000. According to them, quality is defined as follows:

Definition 2 [9] Quality is the totality of features and characteristics of a product or service that bear on its ability to satisfy stated or implied needs.

\section{Quality of services}

According to the stated approach taken for the SQUADRA project, our interest is mainly focused on identifying parameters for benchmarking that can represent quality features. That is [4] [3], parameters that are capable of evaluation against defined standards of acceptability.

Such a capability is the effect of what is called [9] quality policy, that is

Definition 3 The overall quality intentions and directions of an organization as regards quality, as formally expressed by top management

In order to determine and implement the quality policy it is necessary:

1. to know what is produced; this requires to analyze the system in order to identify the produced services;

2. to know how production is performed; this requires to build a model of the system, specifying the production steps needed to provide the services;

3. to decide how good the produced services have to be.

In the present study, which refers to the first phase of the SQUADRA project, we are concerned with the above points 1 and 2 . As it should be evident, they correspond to the phase of analysis of the benchmarking techique.

\section{Results of the first phase of the SQUADRA project}

In this section we present some of the results of the first phase of the SQUADRA project, that appear to be of interest with respect to the modelling activities of the benchmarking technique.

\subsection{Methodological choices}

The cornerstone upon which the project methodology is based is that quality is not an attribute that can be added to the service at the end of its production process; in fact, quality has to be conceived, designed, built, verified and maintained at the same time as the service is conceived, designed, built, verified and maintained.

Such a statement brings the following consequences: 
1. the whole service lifecycle must be taken into account; opposite to the tayloristic, procedure-oriented approaches, which tend to hide business processes, the whole life of the service must be considered

2. analysis must be conceptually approached, and mostly performed in practice, in a top-down way;

the dimension and complexity of the system, and the need to consider its position within its context, require an analysis approach granting:

- the possibility of modelling a system at various detail levels, without losing the globality of the description

- the possibility of concentrating the analysis on specific parts of the system, without missing the relations among them

3. the model of the production cycle of the services must be implementationfree;

the production cycle of the service (which is only a part of its life cycle) must be represented in terms of functional elements in order to provide the widest choice of opportunities to the qualification of the services in terms of quality; this is of crucial importance not only for the SQUADRA project, but also for using models in the analysis, design and measurement phases of the general benchmarking technique

4. the transversality of the steps of the service production process, with respect to the functional (i.e.the vertical) structure of the system, must be emphasized

in this way the complex of the internal elements concurring to the production of the service, and the relations among them, can be appropriately pointed out, as well as the connections between the system and its context, both in input and output

\subsection{Results of the first phase of the SQUADRA project}

\subsubsection{The models of the service production process}

The methodological results of the first phase of the SQUADRA project that bear relevance for the analysis step of the benchmarking technique are:

- use of analysis techniques, in particular the structured analysis of De Marco Yourdon [8];

- use of rules and techniques of graphic representation, in particular the ones of Yourdon;

- use of automatic tools for preparing project reports which have a widespread diffusion and high level of user friendliness, in order to facilitate exchange within both the local projects and the global benchmarking activity. 
The actual activity devoted to the analysis of the system has yielded a number of graphical models, with the features discussed above, that must consitute a solid starting point for the analysis phase of the benchmarking activity.

Owing to the philosophy of the project, an exhaustive and detailed coverage of the central administration of the University was not really important. Rather, assessing the possibilities provided by the adopted techniques was a major point.

Models consist of Data Flow Diagrams (DFD), representing flows involving service production steps and external entities. Service production steps are activities which transform inputs into outputs and, hopefully, add some value to inputs in view of the service to be delivered, with respect its expected quality feature levels. External entities are element interacting with the system providing services, and lie outside the system boundaries. According to the quality-oriented approach of the project, they have been classified into four categories, which are, in order of importance:

- clients: they are the ultimate users of the service;

- activators: they are the external elements that trigger the production of the service; occasionally, they can be the same as the end users of the service, but not necessarily;

- decision units: they are part of the service production process that do not perform any transformation, but can decide on the continuation or interruption of the production process; more specifically, they often have to guarantee the accompliance of the production process to laws and regulations;

- external support units: they provide support services to the internal production process, such as, for instance, banking, mailing, etc.

The first diagram that has been produced is the so called context diagram. It represents the central administration of the University of Trieste, i.e. the system under consideration, at the maximum level of aggregation and all the flows with which it interacts with its environment. Such flows are the ones crossing the boundary of the system we are studying.

The second representation of the system is provided at a more detailed level and four main groups of services are identified, concerning services provided by the central administration to the main categories in which the users can be grouped: students, internal personnel and internal structures such as faculties or departments.

These services are the management of financial resources, of personnel, of buying and selling goods and services, and of what relates to students.

The four processes of this second level have been then analized at increasing levels of disaggregation, thus producing further DFDs.

The model produced has been thoroughly discussed with the Administrative Director of the University and the officers in charge of the Department of the University Administration which provide the services considered. A very good level of agreement has been achieved concerning the correspondence between the processes represented in the model and the functional steps of the actual production 
cycle. Also, it has been verified that, for each service, clients, activators and decision makers have been correctly identified.

In the perspective of benchmarking for the administrative services of Universities, the model formulated in the SQUADRA project can constitute a sound base on which the structure, functionalities and procedures of different organizations can be analyzed and compared. To this end, the possibility of performing verifications and validations based on a formal model is of particular interest, as such activities bear an especially critical relevance for complex organizations.

\section{Conclusions}

We believe that activities devoted to the assessment of organizations providing services must be performed according to a quality-directed approach. In this way the highest benefit may be achieved from these activities in order to devise how to improve the performance of the system.

As a consequence, also the benchmarking techniques adopted for such activities must be used in a quality-oriented way. This requires for the phases of analysis, design of measurements, and measurement, using analysis and modelling techniques for the organization of interest, and for the other ones, having the capability of representing the key elements and factors that characterize a service. Also, having used formal models and automatic representation tools, the continuous updating and improving of the system representation is made much more convenient. Finally, such techniques are simple and easy to understand, in order to facilitate communication both within and between organizations.

We believe that these results of the SQUADRA project constitute a sound conceptual base to perform benchmarking activities among different organizations, in which each of them compares its own performance with the performance of every other one. For this kind of polycentric benchmarking we have also provided a formal methodological approach and practical tools to analize and represent the systems.

\section{References}

[1] Philip B. Crosby. Quality is free. McGraw-Hill, 1979.

[2] W. Edwards Deming. Out of the Crisis. Quality, Productivity and Competitive Position. Cambridge University Press, 1986.

[3] Roger Fournier. Practical Guide to Structured System Development and Maintenance. Yourdon Press, 1991.

[4] EN 29004-2. Quality management and quality system elements - Guidelines for services. 1991.

[5] Henry Mintzberg. Patterns in strategy formation. Management Science 24, May 1978, pp. 934-948. 
[6] Martyn A. Ould. Strategies for software engineering - the management of risk and quality. Wiley, 1990.

[7] Roger Pressman. Software engineering: a practitioner's approach. McGrawHill, 1990.

[8] Edward Yourdon. Modern structured analysis. Prentice-Hall, 1989.

[9] EN 28402. Quality - Terminology. June 1992. 